\title{
Unexpected Hydrodynamic Behavior of Multielectron Bubbles in Liquid Helium I
}

\author{
U. Albrecht and P. Leiderer \\ Fakultät für Physik, Universität Konstanz, Konstanz, Germany
}

(Received August 13, 1991)

We have investigated multielectron bubbles (with charges $\mathrm{Z} \sim 10^{6}$ electrons) in normal liquid ${ }^{4} \mathrm{He}$. The drift velocity in an electric field and the charge of the bubbles were measured simultaneously. We found the drag coefficient to be over an order of magnitude higher than expected from ordinary hydrodynamics. The velocities did not show a correlation with the charge and could vary up to a factor of 3 for equally charged bubbles.

\section{INTRODUCTION}

Liquid helium has a negative electron affinity, therefore its free surface can be charged with electrons (provided that the electron energy is smaller than the energy barrier at the surface of about $1 \mathrm{eV}$ ). In the direction perpendicular to the surface the energy is quantized, the ground state being $8 \mathrm{~K}$ below the vacuum level. Horizontally the electrons are free to move and thus constitute a 2-dimensional Coulomb system. Charging the surface above a critical electron density $n_{c}=2.4 \cdot 10^{9} \mathrm{~cm}^{-2}$ leads to an electrohydrodynamic instability. ${ }^{1}$ The electrons eventually break through the surface, forming macroscopic, charged multielectron bubbles (MEB), which are stabilized by the surface tension and the hydrostatic pressure in the liquid counterbalanced by the Coulomb repulsion of the electrons. The radius of these objects is calculated by minimizing the total energy (neglecting hydrostatic pressure) to

$$
R=\left(\frac{Z^{2} e^{2}}{16 \pi \sigma \varepsilon}\right)^{1 / 3}
$$

(where $Z$ : number of electrons in MEB, $e$ : electron charge, $\sigma$ and $\varepsilon$ : surface tension and dielectric constant of liquid helium respectively). Typical diameters range from 10 to $100 \mu \mathrm{m}$ for charges from $10^{5}$ to $10^{7}$ electrons. The 
electrons inside the MEB are localized about $10 \mathrm{~nm}$ above the bubble surface, forming again a 2D electron system with a now spherical geometry. ${ }^{12}$ Theoretical $^{3-8}$ and experimental ${ }^{2,9,10}$ investigations of MEB have been motivated by the prospect of obtaining 2D electron systems at densities much higher than on the free helium surface, as well as by the peculiar nature of the system itself. Representing a macroscopic, clean electrohydrodynamic system it could be used as a testing ground for theoretical predictions on the dynamics and stability of charged spheres, which are also relevant for the behavior of heavy nuclei. Salomaa and Williams $s^{6,7}$ have made theoretical predictions on the stability of MEB. In a previous publication we reported the observation of oscillations and also of fissioning of the bubbles.'

In this paper we present simultaneous measurements of velocity and charge of MEB. The observed velocities cannot be understood within what is known about ordinary gas bubbles in liquids.

\section{EXPERIMENTAL}

Electrons were emitted from a heated tungsten filament. They were drawn to the helium surface by an electric field applied between the filament and an anode tip immersed in the liquid helium (as shown in Fig. 1). The helium temperature was kept above the lambda point at around $3.5 \mathrm{~K}$.

When reaching the critical conditions MEB developed from the resulting surface instability and travelled downward towards the anode tip through the liquid under the influence of the electric field. The MEB were observed by small angle light scattering (using a $\mathrm{HeNe}$-laser) and recorded on videotape. Each videoframe shows a track corresponding to the distance the MEB travelled during the exposure time $(20 \mathrm{~ms})$. By chopping the laser we could directly measure the bubble speed through the spacing between the illuminated portions of the track. The bubble charges were determined by measuring the displacement current induced in the anode during the passage of the MEB through the (inhomogeneous) electric field. Integration of the displacement current gives the bubble charge. The current signal was recorded simultaneously with the video pictures.

\section{RESULTS}

Figure 1 shows a typical bubble track at chopped illumination copied from the video monitor (Fig. Ib) and the corresponding charge signal (Fig. la). Lines of constant electric potential are indicated. The bubble moves toward the tip with a constant velocity in the region of approximately uniform electric field (we only evaluated the velocities in that region). All observed MEB reach a steady state velocity shortly after leaving the surface, 


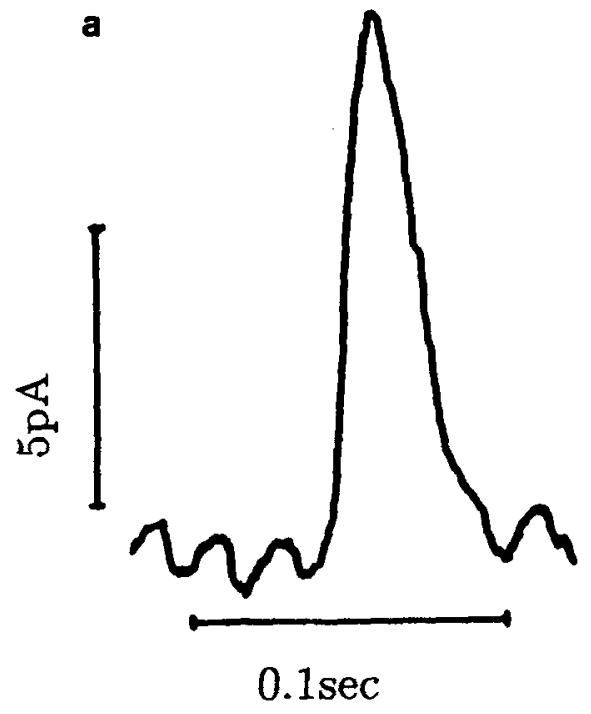

b
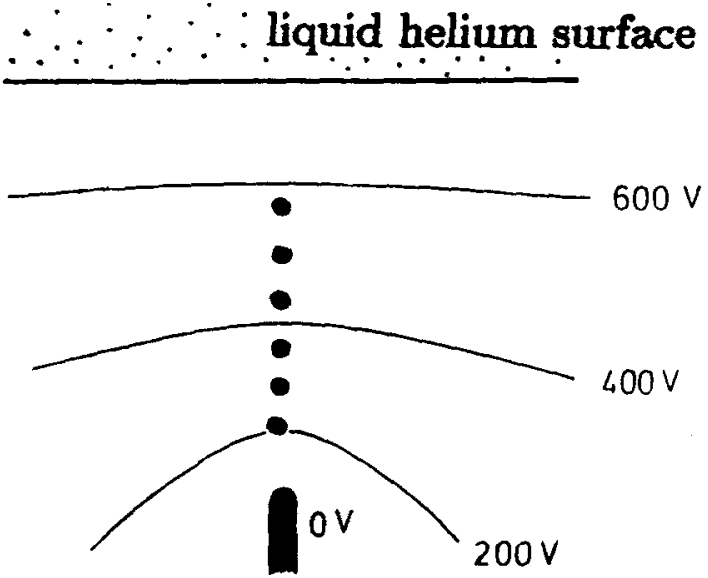

Anode

Fig. 1. (a) Displacement current induced in the anode by motion of a MEB with $Z=1.4 \cdot 10^{6}$ electrons towards it through the liquid helium. (b) shows a sketch of the corresponding videopicture at chopped laser illumination (period $\tau=3.7 \mathrm{~ms}$ ). 
i.e. the force on the bubble exerted by the electric field is in equilibrium with the drag force. Figure 2 shows the measured velocities, which are on the order of a few centimeters per second, versus the charge of the bubbles. The result is surprising, because although we are working with a clean and hydrodynamically very well defined system the velocities scatter well beyond experimental uncertainty even for equally charged MEB, and they do not show any significant dependence on the bubble charge.

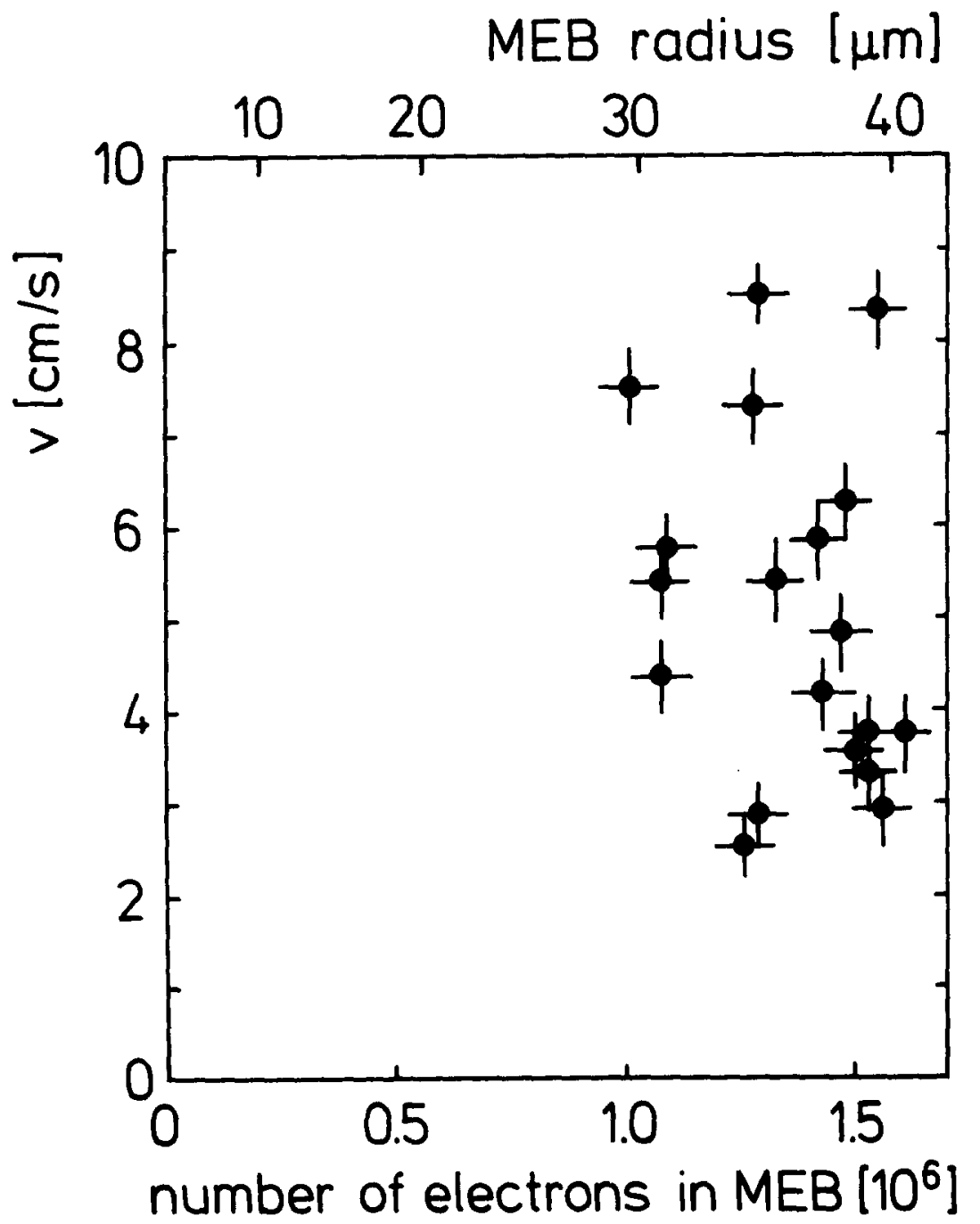

Fig. 2. Measured velocity vs. MEB charge. The calculated equilibrium radii (eq. (1)) are indicated on the upper scale. 
In a simple hydrodynamic treatment for gas bubbles in liquids the velocity should be in first order proportional to the force exerted on it (Stokes law), with corrections due to turbulence and bubble deformation at higher velocities. To describe hydrodynamic behavior it is convenient to define the following dimensionless quantities:

(i) the drag coefficient

$$
c_{w}=\frac{F_{d}}{\pi R^{2} \frac{1}{2} \rho v^{2}}
$$

( $F_{d}:$ drag force, $\rho$ : liquid density, $v$ : velocity) relating the drag force and the normal force, and

(ii) the Reynolds-number

$$
R e=\frac{2 \rho v R}{\eta}
$$

( $\eta$ : viscosity of the liquid) characterizing the influence of turbulent effects. Figure 3 shows schematically the expected dependence between drag coeffcient and Reynolds-number; the solid line summarizes absolute values taken from various measurements on gas bubbles in liquids, subject only to bouyancy. ${ }^{10}$ The simple Stokes law $F_{d}=6 \pi \eta r v$, corresponding to a slope of -1 in the doubly logarithmic plot, is only valid for $\operatorname{Re} \leq 1.5$. The rise of $c_{w}$ around $\operatorname{Re}=10^{2}$ to $10^{3}$ is due to a deformation of the bubbles at higher velocities. Our data are plotted as solid circles. The apparent slope of -2 reflects the fact that the velocity scatters irregularly at nearly constant force and is due to the definitions of $c_{w}$ and $\operatorname{Re}$ (since $c_{w} \sim 1 / v^{2}$ and $\operatorname{Re} \sim v$, a variation of $v$ at contant $F$ leads to $c_{w} \sim 1 / \operatorname{Re}^{2}$ ). A comparison with the expected values shows that the drag coefficient for the MEB is more than an order of magnitude higher than for comparable ordinary gas bubbles in liquids.

\section{DISCUSSION}

As an apparent explanation for the observed results one might suspect that the MEB in our experiment had not reached their equilibrium size given by Eq. (1), e.g. warm He gas being enclosed in the bubble during its formation process at the surface would add to the electronic pressure and thus increase the bubble radius. However, from an analysis of the videopictures we can exclude that they are a lot larger than the equilibrium radii corresponding to the charges measured (see Fig. 1). A second origin for nonequilibrium, loss of electrons by tunneling from MEB into single-electron bubble states, ${ }^{8}$ can also be excluded, since in that case one should observe a significant change in the velocity of the bubble on its way to the anode, which is 


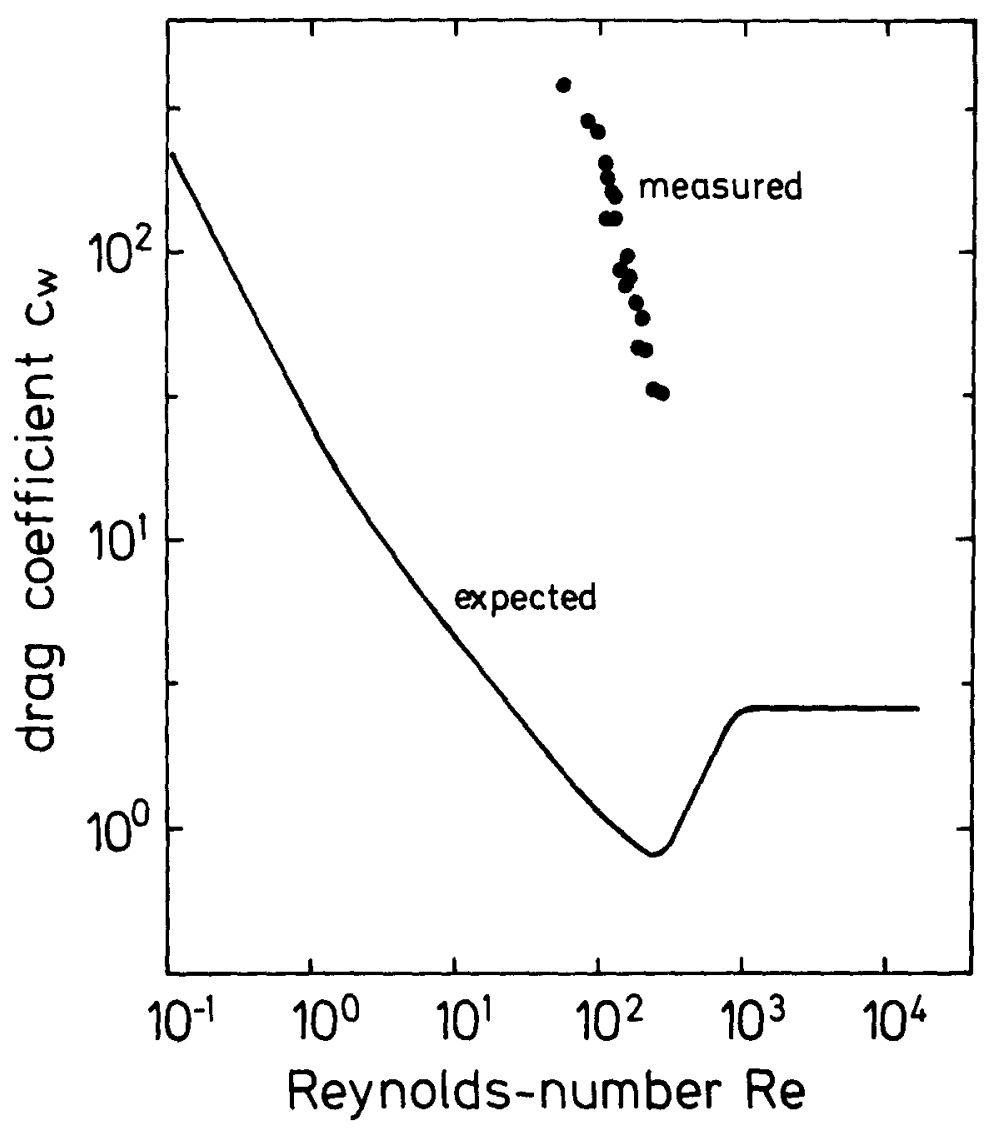

Fig. 3. Drag coefficient vs. Reynolds number as expected for ordinary gas bubbles and as measured for MEB. The slope of -2 for the measured data reflects the large irregular scattering of the bubble velocity at nearly constant force.

not the case (to explain the observed drag coefficient the number of electrons in the bubble would have to decrease by almost two orders of magnitude). Convective currents, which we also have considered, would lead to a correction opposite to the effect observed and can therefore be ruled out as well.

The large discrepancy between the hydrodynamical behavior of MEB and ordinary gas bubbles thus indicates that most of the energy ZeEv (amounting to some $10^{-2} \mathrm{erg} / \mathrm{s}$ ) picked up per second by the bubble in the electric field $E$ is dissipated into the liquid. One way of dissipating bubble energy is through their quite prominent oscillations (with typical frequencies of a few kHz). ${ }^{9}$ Following a calculation of Ceschia and Nabergoj ${ }^{11}$ for the energy dissipation of a stationary oscillating bubble at rest in a viscous liquid 
we get values around $10^{-3} \mathrm{erg} / \mathrm{s}$ for oscillation amplitudes of a few percent of the radius (higher amplitudes should not be stable for MEB ${ }^{6,7}$ ) Nevertheless this mechanism may account at least for part of the observed effects. The motion of the bubble through the liquid, which is not accounted for in the above calculation, can lead to an increased amount of dissipated energy. It can also not be excluded that there is an additional mechanism stabilizing higher oscillation amplitudes (which enter quadratically into the dissipation) than predicted. The excitation of different oscillation modes would naturally explain the large scatter between the velocities. More detailed measurements of the bubble oscillations should allow to test this hypothesis.

\section{SUMMARY}

MEB show a hydrodynamic behavior very distinct from ordinary gas bubbles in liquids. Their velocity in an electric field does not depend significantly on the electrostatic force exerted on them and shows a large scatter well beyond the experimental uncertainty. They experience a drag over an order of magnitude higher than that expected for ordinary gas bubbles. We attribute these effects to energy dissipation caused by oscillations of the MEB.

\section{ACKNOWLEDGMENTS}

Helpful discussions with V. B. Shikin and J. Jäckle are gratefully appreciated.

\section{NOTE ADDED IN PROOF}

Salomaa et al. have carried out a more detailed calculation of the electronic structure of MEB and the dependence of their radius on temperature and pressure. M. M. Salomaa, P. I. Soininen, S. T. Hannahs, and G. A. Williams, (manuscript in preparation).

\section{REFERENCES}

1. L. P. Gorkov and D. M. Chernikova, JETP Lett. 18, 68 (1973); P. Leiderer, Physica B+C 126, $92(1985)$.

2. A. P. Volodin, M. S. Khaikin and V. S. Edelman, JETP Lett. 26, 543 (1977); M. S. Khaikin, J. Phys. Colloq. C6, 1295 (1978).

3. V. B. Shikin, JETP Lett. 27, 39 (1978).

4. M. M. Salomaa and G. A. Williams, Phys,. Rev. Lett. 47, 1730 (1981).

5. E. A. Manykin, M. I. Ozhoven and P. P. Poluétkov, Sov. J. Low Temp. Phys. 8, 215 (1982).

6. M. M. Salomaa, G. A. Williams, Phys. Scr. T4, 204 (1983). 
7. S. T. Hannahs, G. A. Williams, M. M. Salomaa, in Proceedings of the XVII International Conference on Low Temperature Physics, ed. U. Eckern et al. (North-Holland, Amsterdam) 1984, p. 297.

8. A. A. Artem'ev, A. G. Khrapak and I. T. Yabukov, Sov. J, Low Temp. Phys. 11, 555 (1985).

9. U. Albrecht and P. Leiderer, Europhys. Lett. 3, 705 (1987); U. Albrecht, P. Leiderer, Can. J. Phys. 65, 1536 (1987).

10. B. T. Chao, Phys. Fluids (USA) 5, 69 (1962); E. Coester, Z. Angew. Phys. 13, 254 (1961); I. Yaron, B. Gal-Or, Int. J. Heat Mass Tranfer 16, 887 (1973).

11. M. Ceschia and R. Nabergoj, Phys. Fluids (USA) 21, 140 (1978). 